\title{
Author Correction: Dynamically induced cascading failures in power grids
}

\author{
Benjamin Schäfer (10) ${ }^{1,2}$, Dirk Witthaut (ib) ${ }^{3,4}$, Marc Timme ${ }^{1,2}$ \& Vito Latora (i) ${ }^{5,6}$
}

Correction to: Nature Communications https://doi.org/10.1038/s41467-018-04287-5; published online 17 May 2018

The original version of this Article omitted the following from the Acknowledgements:

'Finally, we gratefully acknowledge support from the German Science Foundation (DFG) by a grant toward the Cluster of Excellence "Center for Advancing Electronics Dresden” (cfaed)"

This has been corrected in both the PDF and HTML versions of the Article.

Published online: 27 September 2018

\begin{abstract}
(c) Open Access This article is licensed under a Creative Commons Attribution 4.0 International License, which permits use, sharing, adaptation, distribution and reproduction in any medium or format, as long as you give appropriate credit to the original author(s) and the source, provide a link to the Creative Commons license, and indicate if changes were made. The images or other third party material in this article are included in the article's Creative Commons license, unless indicated otherwise in a credit line to the material. If material is not included in the article's Creative Commons license and your intended use is not permitted by statutory regulation or exceeds the permitted use, you will need to obtain permission directly from the copyright holder. To view a copy of this license, visit http://creativecommons.org/licenses/by/4.0/.
\end{abstract}

(C) The Author(s) 2018

\footnotetext{
${ }^{1}$ Chair for Network Dynamics, Center for Advancing Electronics Dresden (cfaed) and Institute for Theoretical Physics, Technical University of Dresden, 01062 Dresden, Germany. ${ }^{2}$ Network Dynamics, Max Planck Institute for Dynamics and Self-Organization (MPIDS), 37077 Göttingen, Germany.

${ }^{3}$ Forschungszentrum Jülich, Institute for Energy and Climate Research - Systems Analysis and Technology Evaluation (IEK-STE), 52428 Jülich, Germany. ${ }^{4}$ Institute for Theoretical Physics, University of Cologne, 50937 Köln, Germany. ${ }^{5}$ School of Mathematical Sciences, Queen Mary University of London, London E1 4NS, UK. ${ }^{6}$ Dipartimento di Fisica ed Astronomia, Università di Catania and INFN, 95123 Catania, Italy. Correspondence and requests for materials should be addressed to B.Säf. (email: benjamin.schaefer@tu-dresden.de) or to M.T. (email: marc.timme@tu-dresden.de) or to V.L. (email: v.latora@qmul.ac.uk)
} 